\title{
An Account of the Tribe Eragrosteae of Japan. I.
}

\author{
By
}

\section{Jisaburo Ohwi.*}

Received April 2, 1941.

Gen. 1. Eragrostis Beauv. Ess. Agrost. (1812) 70.-Typus : Eragrostis Eragrostis (Linn.) Beauv. (E. poaeoides).

1. Spiculae minus quam $2 \mathrm{~mm}$ longae, lemma $0.7-0.8 \mathrm{~mm}$ longum, antherae $1 / 4-1 / 6$ lemmatis aequilongae.

2. Paleae carina longe ciliata, ciliis $1 / 2$ latitudine lemmatis aequilongia, culmi basi

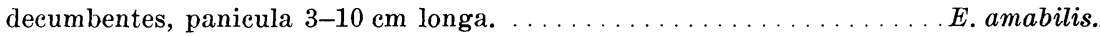

2. Paleae carina brevissime ciliata scabra, culmi erecti, panicula angusta, $5-60 \mathrm{~cm}$

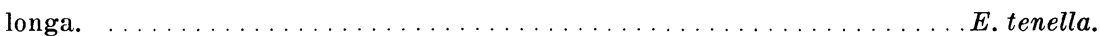

1. Spiculae plus quam $2 \mathrm{~mm}$ longae, lemma longius quam $1 \mathrm{~mm}$.

2. Spiculae valde compressae ovatae 10-20-florae pallidae et saepe rubro-suffusae, lemma patentissimum, cum palea facile deciduum, antherae $1 / 6-^{1} / ; \mathrm{mm}$ longae.

E. unioloides.

2. Spiculae plus minus compressae lanceolatae vel lineares.

3. Spiculae, pedicelli, folia subtus ad costas, vaginae, ete. saltem partim glandulis sessilibus vel impressis obsitae.

4. Antherae $1 / 4-1 / 6 \mathrm{~mm}$ longae, lemma acutum vel subobtusum.

5. Spiculae $2.5 \mathrm{~mm}$ latae, lemma et gluma ad carinas glandulosa.

E. megastachya.

5. Spiculae 1.5-2 mm latae, lemma et gluma eglandulosa. . . . . E. poaeoides. 4. Antherae $0.8-1 \mathrm{~mm}$ longae, lemma purpurascens, eum glumis eglandulosum. .

3. Planta omnibus partibus eglandulosa.

E. ferruginea.

4. Panicula angusta dense spiculosa spiciformis, antherae $1 / 3 \mathrm{~mm}$ longae.

5. Panicula densissima pallide virens vel stramineo-virens, ramis axilla pilosis, paleae carina longe ciliata. .................... cylindrica.

5. Panicula densa, saepe plus purpurascens, ramis axilla nudis, paleae carina brevi-ciliata.

E. Nevinii.

4. Panicula laxa, non spiciformis.

5. Spiculae minus quam $1.5 \mathrm{~mm}$ latae, $3-5 \mathrm{~mm}$ longae, antherae $1 / 4 \mathrm{~mm}$ longae, planta annua.

6. Vaginae apice, rami paniculae axillaque longe pilosae, planta saepe

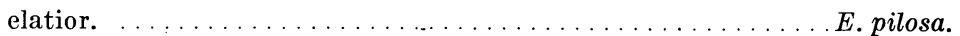

6. Vaginae apice rami paniculaeque undae. . . ........ Eulticaulis.

๊. Spiculae latiores quam $1.5 \mathrm{~mm}, 5-20 \mathrm{~mm}$ longae, planta perennis.

6. Antherae $0.5-0.7 \mathrm{~mm}$ longae.

7. Nervi laterales lemmatis prominuli, laminae foliorum glabrae vel parce longe-pilosae, caryopsis elliptica, cellulis extimis obsoletis.

8. Palea cum lemmate decidua, caryopsis vix compressa oblonga. ....

E.Chariis.

* Botanical Institute, Kyoto Imperial University, Kyoto. 
8. Palea diu persistens, lemma decidnum, caryopsis elliptica, a latere

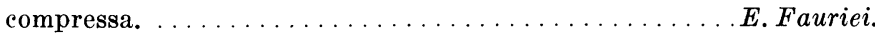

7. Nervi laterales lemmatis vix prominentes, laminae pilosae, caryopsis late ovoidea obtuse trigona, cellulis extimis reticulatis. E. pilosissima. 6. Antherae minus quam $1 / 3 \mathrm{~mm}$ longae, caryopsis elliptica.

7. Spiculae pallidae vel olivaceae non purpurascentes, laterales ascendentes vel patentes, lemma 1.5-2 mm longum acutum vel obtusulum.

8. Spiculae pallidae vel straminescentes, laterales sessiles vel brevissime pedicellatae patentes, lemma apice obtusulum et saepe mucronulatum, paniculae rami ramulique saltem juvenile pilis longis patentibus parcissime obsiti. ............. pilosiuscula.

8. Spiculae olivaceo-tinctae, laterales vulgo brevi- vel longiuscule-pedicellatae ascendentes, lemma acutum, paniculae rami ramulique

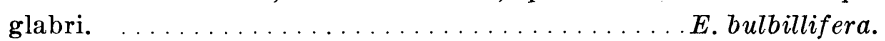

7. Spiculae cinerascente-rubro-purpureae, laterales brevi-pedicellatae adpressae, lemma acutum dorso conspicue carinatum, $1.5 \mathrm{~mm}$ longum.

E. aquatica.

Sp. 1. Eragrostis amabilis (Linn.) Wight et Arn. ex Hook. et Arn. Bot. Beech. Voy. (1841) 251; Hiтchc. in Lingn. Sci. Journ. 7, 1929 (1931) 190 et Man. Grasses U.S. (1935) 147 et 847.

Poa amabilis Lrnn. Sp. Pl. 1753) 68.

Poa plumosa Retz. Obs. Bot. 4 (1786) 20.

Eragrostis plumosa (RETz.) Link, Hort. Berol. 1 (1827) 192 ; HAck. in Bull. Herb. Boiss. 7 (1899) 724; Honda, Monogr. Poac. Japon. (1930) 109.

Nom. Jap. Nuka-kazekusa.

Hab. Ins. Riukiu (Ishigaki, Iriomote), Formosa.

Sp. 2. Eragrostis tenella (Linn.) Beauv. ex Roem. et Schult. Syst. 2 (1817) 576; Franch. et Savat. Enum. Plant. Japon. 2 (1879) 177 ; Hitchc. Man. Gr. U.S. (1935) 168 et 853.

Poa tenella Linn. Sp. Pl. (1753) 69.

Poa japonica Thunb. Fl. Jap. (1784) 51.

Eragrostis japonica (Thunb.) Trin. in Mém. Acad. St. Pétersb. 6:1 (1830) 108; Hiтchс. in Lingn. Sci. Journ. 7 (1931) 191; Hack. in Bull. Herb. Boiss. 7 (1899) 705; Jedw. in Bot. Arch. Mez. 5 (1924) 180; Honda, Monogr. Poac. Japon. (1930) 108.

Eragrostis aurea STEUd. Synops. 1 (1854) 267.

Eragrostis tenella var. japonica (Thunb.) Roem. et Schult. Syst. 2 (1817) 576.

Nom. Jap. Kogome-kazekusa.

Hab. Shikoku, Kiushiu, Korea australis, Riukiu, et Formosa.

Sp. 3. Eragrostis unioloides (Retz.) NeEs ex Steud. Synops. 1 (1854) 
264 ; Hack. in Bull. Herb. Boiss. 7 (1899) 724; Honda, Monogr. Poac. Japon. (1930) 107; Hiтchc. Man. Gr. U.S. (1935) 149 et 853.

Poa unioloides Retz. Observ. Bot. 5 (1789) 19.

Eragrostis amabilis (non Hook. et ArN.) Hook. f. Fl. Brit. Ind. 7 (1897) 317; Rendle in Journ. Linn. Soc. 36 (1904) 412; Hayata, Icon. Pl. Formos. 7 (1918) 92.

Eragrostis formosana Hayata in Bot. Mag. Tokyo 21 (1907) 53, et Mat. Fl. Formos. (1911) 408.

Nom. Jap. Beni-suzumegaya.

Hab. Formosa.

Sp. 4. Eragrostis megastachya (Koel.) Link, Hort. Berol. 1 (1827) 187 ; Franch. et Savat. Enum. Plant. Japon. 2 (1879) 177; Aschers. et Graebn. Synops. $2: 1$ (1900) 370; Honda; Monogr. Poac. Japon. (1930) 105 ; Roshev. in Komar. Fl. URSS. 2 (1934) 316.

Briza Eragrostis Linn. Sp. Plant. (1753) 70.

Poa megastachya Koel. Descr. Gram. (1802) 181.

Eragrostis major Hos'r. Icon. Gram. Austr. 4 (1809) 14.

Eragrostis cilianensis Link ex VIGN. Lut. in Malpighia 18 (1904) 386, cfr. Mansf. in Fedde Repert: 49 (1940) 43; Hitrhc. Man. Gr. U.S. (1935) 154 et 848 .

Nom. Jap. Suzumegaya.

Hab. Hondo, Shikoku, Kiushiu, Korea, Riukiu, Formosa.

Sp. 5. Eragrostis poaeoides Beauv. Ess. Agrost. (1812) 162, nom. nud.; Roem. et Schult. System. 2 (1817) 574; Hitchc. Man. Gr. U.S. (1935) 156 et 851; Mansf. in Fedde Repert. 45 (1938) 224; Kitagawa, Lin. Fl. Mansh. (1939) 76.

Poa Eragrostis Linn. Sp. Plant. (1753) 68.

Eragrostis minor Host: Icon. Gram. Austr. 4 (1809) 15, nom. illeg.; Hack. in Bull. Herb. Boiss. 7 (1899) 724 ; Aschers. et Graebn. Synops. $2: 1$ (1900) 372; Jedw. in Bot. Arch. Mez. 5 (1924) 210; Honda, Mon. Poac. Japon. (1930) 106.

Eragrostis Eragrostis (Linn.) Beauv. l. c. (1812) 71 et 174.

Nom. Jap. Ko-suzumegaya.

Hab. Honda, Shikoku, Kiushiu, Korea, Riukiu, et Formosa.

Sp. 6. Eragrostis ferruginea (Thunb.) Beauv. Ess. Agrost. (1812) 71; Steud. Synops. 1 (1854) 267; Jedw. in Bot. Arch. Mez 5 (1924) 194 ; Honda, Monogr. Poac. Jap. (1930) 104 ; Kitagawa, Lin. Fl. Mansh. (1939) 75 .

Poa ferruginea Thunв. Fl. Jap. (1784) 50. 
Poa barbata Thunb. Fl. Jap. (1784) 50, t. 10.

Eragrostis orientalis Trin. in Bunge, Enum. Pl. Chin. Bor. (1831) 71.

Eragrostis pogonia S.TEUD. Synops. 1 (1854) 267.

Eragrostis Thunbergii Koldzumi in Bot. Mag. Tokyo 39 (1925) 304.

Nom. Jap. Kazekusa.

Hab. Hondo, Shikoku, Kiushiu, Korea.

Sp. 7. Eragrostis cylindrica (Roxb.) Nees ex Hook. et Arn. Bot. Beech. Voy. (1841) 251; Hitcirc. in Lingn. Sci. Journ. 7 (1931) 192; OHw in Journ. Japan. Bot. 13 (1937) 444.

Poa cylindrica RoxB. Fl. Ind. 1 (1820) 335.

Eragrostis geniculata Nees et Mey. in Nov. Act. Acad. Caes. Leop. Carol. 19, suppl. 1 (1841) 71; Cam. in Lecomte, Fl. Indochin. $7: 5$ (1922) 560 p.p.; Honda, Monogr. Poac. Japon. (1930) 109.

Eragrostis lobata (non Trin.) Jedw. in Bot. Arch. Mez 5 (1924) 179.

Nom. Jap. Hizaori-numeri.

Hab. Formosa.

Sp. 8. Eragrostis Nevinii Hance in Journ. Bot. 18 (1880) 302 : Hiтchc. in Lingn. Sci. Journ. 7 (1931) 192 ; OHwi, l. c. 444.

Nom. Jap. Ho-suzumegaya.

Hab. Formosa.

Sp. 9. Eragrostis pilosa (Linn.) Beauv. Ess. Agrost. (1812) 71, 162, et 175 ; Miq. Prol. Fl. Japon. (1867) 168 ex pte; HAck. in Bull. Herb. Boiss. 7 (1899) 706 et $2: 3$ (1903) 505; Jedw. 1. c. 208, ex pte; Honda, Monogr. Poac. Jap. (1930) 102 ; Hitchc. Man. Gr. U.S. (1935) 150 et 851 ; KitaGawa, Lin. Fl. Mansh. (1939) 76. ${ }^{*}$

Poa pilosa Linn. Sp. Pl. (1753) 68.

Nom. Jap. O-niwahokori.

Hab. Hondo, Kiushiu, Shikoku, Korea, Riukiu, Formosa.

Sp. 10. Eragrostis multicaulis Steud. Synops. 1 (1854) 426; OHw in Journ. Jap. Bot. 13 (1937) 445.

Glyceria airoides STEUd. Synops. 1 (1854) 287.

?Eragrostis pilosa var. nana Miq. Prol. Fl. Jap. (1867) 168.

Eragrostis pilosa var. imberbis Franch. Pl. David. 1, in Nouv. Arch. Mus. Paris, $2: 7$ (1884) 145.

* Eragrostis pilosa BeAuv. var. jeholensis (HoNDA) OHWI, comb. nov.-Eragrostis jeholensis Honda in Rep. First Sci. Exp. Manch. 4: 2 (1935) 6, t. 1; Kitagawa, l.c. 75.-Differt a typo spiculis 5-7floris obscurius purpurascentibus.-Hab. Manchuria, China bor. 
Eragrostis pilosa ('vix Beauv.) Auct. fl. Jap. p.p. etiam Jedw. l. c. 208, ex pte.

Eragrostis Niwahokori Honda in Bot. Mag. Tokyo 41 (1927) 387 et Monogr. Poae. Jap. (1930) 101; Miyabe et Kudo, Fl. Hokk. a. Saghal. 2 (1931) 153.

Eragrostis peregrina WiEgand in Rhodora 19 (1917) 95.

Eragrostis pilosa var. condensata $\mathrm{H}_{\mathrm{ACK}}$ in Allg. Bot. Zeitschr. 7 (1901) 13.

Eragrostis damiensiana Ed. Bonnet ex Thell. in Fedde Repert. 24 (1928) 323.

Eragrostis pilosa var. damiensiana ED. Bonnet in Naturaliste 3 (1881) 412.

Nom. Jap. Niwahokori.

Hab. Saghalien. Yezo, Hondo, Shikoku, Kiushiu, Riukiu, Formosa, Korea.

Sp. 11. Eragrostis Chariis (Schultes) Hitcuc. in Lingn. Sci. Journ. 7 (1931) 193 et Marr. Gr. U.S. (1935) 166 et 848.

Poa Chariis Schult. Mant. 2 (1824) 314.

Poa elegans (non Porr.) Roxв. Fl. Ind. 1 (1820) 339.

Poa elegantula Kunth, Rév. Gram. 1 (1829) 114.

Eragrostis elegantula (Kunth) Nees ex Steud. Synops. 1 (1854) 266 ; JeDw..in Bot. Arch. Mez 5 (1924) 191, p.p., non NeEs 1851.

Eragrostis atrovirens (non Trin.) HACK. in Bull. Herb. Boiss. 2:4 (1904) 529, ex pte.

Eragrostis perennans Keng in Sunyatsenia 3 (1935) 16.

Nom. Jap. Kuro-kazekusa.

Hab. Formosa.

Sp. 12. Eragrostis Fauriei Oнwі, sp. nov.

Perennis dense caespitosa, culmis $30-60 \mathrm{~cm}$ altis erectis glabris, foliorum vaginis quam internodiis brevioribus praeter ore longe ciliata glabris, laminis planiusculis elongatis coriaceis $2-5 \mathrm{~mm}$ latis margine involutis, subtus glabris, supra interdum longe sparsim pilosis, panicula exserta 10-15 cm longa laxiuscula, ramis ascendentibus scabris, axilla nudis, spiculis lanceolatis modice compressis $5-10 \mathrm{~mm}$ longis $2.5 \mathrm{~mm}$ latis subdense 6-15-floris ascendentibus vel leviter adpressis distincte pedicellatis griseopallidis vel leviter purpurascentibus, glumis sterilibus late lanceolatis acuminatis uninervibus 1-1.5:1.5-1.7 mm longis, lemmatibus $2 \mathrm{~mm}$ longis ovatis acutissimis minute punctulatis, carina distincta scabris, nervis lateralibus distinctis subparallelis, $\%$ lemmatis percurrentibus, palea 
persistente, carinis arcuatis ciliato-scabris, rhachilla persistente leviter ancipiti, staminibus 3, antheris oblongis atropurpureis $1 / 2 \mathrm{~mm}$ longis, caryopside elliptica leviter compressa obsolete striolata $2 / 3 \mathrm{~mm}$ longa.Ab $E$. Chariis, foliis latis, paleis persistentibus differt.

Nom. Jap. Hiroha-kazekusa.

Hab. Formosa (in montibus Shinten, leg. U. FaUrie n. 153).

Sp. 13. Eragrostis pilosissima Link, Hort. Berol. 1 (1827) 189; Steud. Synops. 1 (1854) 280; Honda, Monogr. Poac. Japon. (1930) 103: Hiтchc. in Lingn. Sci. Journ. 7 (1931) 193.

Poa pilosissima (Link) Kunth, Rév. Gram. 1 (1829) 112 et Enum. Pl. 1 (1833) 330.

Eragrostis Makinoi HaCk. in Bull. Herb. Boiss. 7 (1899) 725 ; Matsum. Ind. Pl. Japon. $2: 1$ (1905) 54.

Eragrostis Millettii Nees in Hook. et Arn. Bot. Beech. Voy. (1841) 252 ; Jedw. in Bot. Arch. Mez. 5 (1924) 185.

Nom. Jap. Hime suzumegaya.

Hab. Riukiu (fide JEDw. l. c.), Formosa.

Sp. 14. Eragrostis pilosiuscula Oнw, sp. nov.

Eragrostis elongata (non JACQ.) Auct. Fl. Formos.

Perennis (vel annua?) caespitosa estolonifera $20-40 \mathrm{~cm}$ alta, culmis erectis glabris tenuibus 1-2-nodis, foliis planis vel laxe involutis 1.5-2.5 $\mathrm{mm}$ latis utrinque sparsim longe hirtis $3-10 \mathrm{~cm}$ longis, ligula brevissima ciliolata, vaginis superne praesertim in ore hirtis, panicula erecta longe exserta $10-15 \mathrm{~cm}$ longa, ramis horizontaliter patentibus solitariis ad $4 \mathrm{~cm}$ longis fere a basi ramulosis, axilla sparse hirta, ramulis abbreviatis fasciculatim 2-5-spiculosis scabriusculis et ramisque saltem juvenile pilis longis patentibus parcissime obsitis, spiculis subsessilibus vel brevissime pedicellatis straminescente pallidis compressis $1.5 \mathrm{~mm}$ latis $6-8 \mathrm{~mm}$ longis $15-20$ floris, glumis late lanceolatis acutis uninervis, lemmatibus late ovatis prominente trinervis ca. $1.7 \mathrm{~mm}$ longis obtusulis et obsolete mucronatis. palea persistente $1.2 \mathrm{~mm}$ longa superne conspicue ciliata, inferne scabra, staminibus 3, antheris atropurpureis ellipticis ca. 1/6 mm longis, caryopside oblonga subcompressa $0.4 \mathrm{~mm}$ longa.-Ab E. elongata (WILLD.) JACQ. differt, paniculae ramis pedicellisque saltem juvenile sparsim longe patentim pilosis, lemmate obsolete mucronato, nervo laterali distinctiore, paleae carinis distinctius ciliatis.

Nom. Jap. Naga-suzumegaya.

Hab. Formosa (Shinchiku, leg.. Y. Shimada n. 4789). 
Sp. 15. Eragrostis bulbillifera Steud. Synops. 1 (1854) 267 ; HACK. in Bull. Herb. Boiss. 7 (1899) 705 et 2:4 (1904) 529.

Eragrostis stenophylla (non Hochst.) JEDw. in Bot. Arch. Mez 5 (1924) 190, quoad syn. p.p.

Eragrostis atrovirens (non Trin.) HACK. 1. c. $2: 4$ (1904) 529, ex pte; Honda, Monogr. Poac. Japon. (1930) 100, ex pte.

Eragrostis elegantula (non NeEs ex StTEUd.) JeDw. l. c. 191, p.p.

Eragrostis Brownii (non NeEs) Miq. Prol. Fl. Jap. (1867) 169.

Eragrostis bahiensis (non Schrad.) Franch. et Savat. Enum. Pl. Japon. 2 (1879) 177.

Eragrostis atrovirens var. ramosa HoNDA in Bot. Mag. Tokyo 45 (1931) 298.

Nom. Jap. Ito-suzumegaya.

Hab. Hondo occident., Shikoku, Kiushiu, Riukiu, Formosa.

Sp. 16. Eragrostis aquatica Honda in Bot. Mag. Tokyo 42 (1928) 508 et Monogr. Poac. Japon. (1930) 99.

Nom. Jap. Numa-kazekusa.

Hab. Hondo occid.

\section{本邦ノすぶめがや族（和交摘要）}

$$
\text { 大 井 次 三 郎 }
$$

Eragrosteae 六原來熱帶ヨ鄉土トシ 本邦デ八臺灣二最モ 種類ガ多ク，舊日本二八 固有種ハアルガ種類ハ餘リ多イト云フ程デハナイ。本邦全體デハ 28 種ガ認メラレ, 歐文闌ノ樣二分類サレル。此族デハ䒬ノ長サ卜頴果ノ形及ビソノ表面ノ玟紋ガ分類 上重要ナ特䡓ノーツトシテ認メラレル。 